\title{
ESTIMATES OF SOLUTIONS \\ TO LINEAR ELLIPTIC SYSTEMS AND EQUATIONS
}

\author{
HEINRICH BEGEHR \\ I. Mathematisches Institut, Freie Universität Berlin \\ Arnimallee 3, D-1000 Berlin 33, Germany
}

Dedicated to the memory of V. S. Vinogradov

Whenever nonlinear problems have to be solved through approximation methods by solving related linear problems a priori estimates are very useful. In the following this kind of estimates are presented for a variety of equations related to generalized first order Beltrami systems in the plane and for second order elliptic equations in $\mathbb{R}^{m}$. Different types of boundary value problems are considered. For Beltrami systems these are the Riemann-Hilbert, the Riemann and the Poincaré problem, while for elliptic equations the Dirichlet problem as well as entire solutions are involved.

1. Introduction. The simplest first order elliptic system in the plane is the Cauchy-Riemann system, in complex form given as

$$
w_{\bar{z}}=0, \quad w=u+i v, \quad z=x+i y, \quad \frac{\partial}{\partial \bar{z}}=\frac{1}{2}\left(\frac{\partial}{\partial x}+i \frac{\partial}{\partial y}\right) .
$$

Its solutions are the analytic functions, the real and imaginary part of which are harmonic, i.e. solutions to the second order elliptic equation

$$
\Delta u=0, \quad \Delta=\frac{\partial^{2}}{\partial x^{2}}+\frac{\partial^{2}}{\partial y^{2}} .
$$

Both equations are very simple and the theories of their solutions are very rich and beautiful. They are special cases of more complicated equations, namely the generalized Beltrami equation

$$
w_{\bar{z}}+q_{1} w_{z}+q_{2} \overline{w_{z}}+a w+b \bar{w}+c=0
$$


and the general second order equation

$$
\sum_{j, k=1}^{2} a_{j k} u_{x_{j} x_{k}}+\sum_{j=1}^{2} b_{j} u_{x_{j}}+c u+d=0, \quad x_{1}=x, x_{2}=y,
$$

respectively. The ellipticity condition for (3) is

$$
\left|q_{1}(z)\right|+\left|q_{2}(z)\right| \leq q_{0}<1,
$$

while (4) is elliptic if $\left(a_{j k}\right)$ is a symmetric matrix and for some positive $\lambda$

$$
\lambda \sum_{j=1}^{2} \xi_{j}^{2} \leq \sum_{j, k=1}^{2} a_{j k} \xi_{j} \xi_{k} \quad \text { for all }\left(\xi_{1}, \xi_{2}\right) .
$$

Rather than considering (4) under (6) with two variables the corresponding equation with $m$ independent variables will be studied.

Several special cases of (3) play an important role in complex analysis. If all the coefficients are zero except $q_{1}$ then (3) is the Beltrami equation, the solutions of which are known as quasiconformal mappings. Their theory became very important for complex analysis and has an interesting counterpart in $\mathbb{R}^{m}$. For $q_{1}=q_{2}=c=0$ equation (3) is the basic equation for generalized analytic functions [51] and for pseudoanalytic functions [22], depending on the assumptions on the coefficients $a$ and $b$. (3) was generalized in different directions. Without going into details we mention the works of Douglis [37], Gilbert-Hile [42, 43] and others, e.g. [6-9], [41], Bojarski [31], Delanghe and co-workers [34], Xu [61].

A variety of boundary value problems were studied in the past for analytic functions. They originated from suggestions by Riemann, and some were first solved by Hilbert and his student Haseman (see e.g. [3]) Hilbert even considered (3) with $q_{1}=q_{2}=c=0$. Russian mathematicians, especially Gakhov [39] and Muskhelishvili [48] developed the theory of boundary value problems for analytic functions cosiderably. Mainly I. N. Vekua and his students, e.g. B. Bojarski, A. Dzhuraev, E. Obolashvili and V. S. Vinogradov, and W. Haak and his group, G. Hellwig, J. Jaenicke, W. Wendland and others extended these problems to more general systems (see $[38,44,47,51,56,59]$ ). In the following three basic boundary value problems for (3) will be presented: the Riemann-Hilbert, the Poincaré and the Riemann boundary value problem. The last problem is sometimes also called the problem of linear conjugacy. There is an immense literature on this subject (see e.g. $[2,3,44,50,51,56,59]$ ) which cannot be quoted here.

The same is true of course for second order elliptic equations, where the literature is even more extensive. But there are a lot of well known books on this subject from which here mainly [40] is used. For second order equations two problems are considered here: the Dirichlet problem for bounded domains and entire solutions, i.e. solutions on the whole space $\mathbb{R}^{m}$ under special growth conditions.

How these estimates for linear problems can be used to treat related nonlinear equations as well as nonlinear boundary conditions can be found e.g. in $[11,16$, 
18, 21]. The method used is a combination of the Schauder imbedding method with the Newton approximation method. This procedure was in this context first applied by Wendland [57] to a quasilinear elliptic first order system in the plane.

2. Riemann-Hilbert problem for generalized Beltrami systems. Let for simplicity $D$ be a bounded simply connected domain in the complex plane $\mathbb{C}$ with continuously differentiable boundary $\partial D$. For the case of multiply connected domains see [56]. For given Hölder continuous functions $\log \lambda$ and $\varphi$ on the boundary $\partial D$, where $\lambda(z) \neq 0$, we look for a solution to

$$
w_{\bar{z}}+q_{1} w_{z}+q_{2} \overline{w_{z}}+a w+b \bar{w}+c=0,
$$

where

$$
\left|q_{1}(z)\right|+\left|q_{2}(z)\right| \leq q_{0}<1
$$

and $a, b, c \in L_{p}(\bar{D})$ for $2<p$, satisfying the boundary condition

$$
\operatorname{Re}\{\overline{\lambda(z)} w(z)\}=\varphi(z), \quad z \in \partial D .
$$

The solutions depend on the index

$$
n=\frac{1}{2 \pi i} \int_{\partial D} d \log \lambda(z)
$$

of the problem, which by continuity is an integer number. As is known from analytic functions theory the homogeneous problem (7) with $\varphi=0$ for nonnegative index has $n$ linearly independent solutions. The general solution becomes unique by imposing side conditions. One possibility is to prescribe

$$
\begin{gathered}
\int_{\partial D} \operatorname{Im}\{\overline{\lambda(z)} w(z)\}|d z|=\kappa, \quad \kappa \in \mathbb{R}, \\
w\left(z_{k}\right)=a_{k}, \quad z_{k} \in D, \quad a_{k} \in \mathbb{C}, \quad 1 \leq k \leq n,
\end{gathered}
$$

for given points $z_{k}$ and values $\kappa, a_{k}$; another is to fix for given points $z_{k}$ on $\partial D$ and $b_{k} \in \mathbb{R}$

$$
\operatorname{Im}\left\{\overline{\lambda\left(z_{k}\right)} w\left(z_{k}\right)\right\}=b_{k}, \quad 0 \leq k \leq 2 n .
$$

For negative index $\varphi$ has to satisfy some conditions in order that (7) be solvable. In the case where $D$ is the unit disc $\mathbb{D}$ these conditions are handled by replacing $\varphi$ by $\varphi+h$ where $h$ has the form

$$
h(z)=\sum_{k=n+1}^{-n-1} h_{k} z^{k}, \quad h_{-k}=\overline{h_{k}}(|k| \leq-n-1),
$$

with coefficients to be determined properly so that the mentioned conditions are satisfied. Hence, in this modified problem besides the solution $w$ the coefficients $h_{k}, 0 \leq k \leq-n-1$, have to be found (see $[51,54,56]$ ). For more general domains $D$, the $z$ on the right hand side of (11) has to be replaced by a conformal mapping from $D$ onto $\mathbb{D}$. 
Basic tools in the theory of generalized Beltrami systems in the plane are two integral operators introduced by I. N. Vekua in his treatment of generalized analytic functions [51],

$$
T \varphi(z):=-\frac{1}{\pi} \int_{D} \varphi(\zeta) \frac{d \zeta}{\zeta-z}, \quad \Pi \varphi(z):=-\frac{1}{\pi} \int_{D} \varphi(\zeta) \frac{d \xi d \eta}{(\zeta-z)^{2}} \quad(\zeta=\xi+i \eta) .
$$

An a priori estimate for solutions to equation (3) was first given in [28] by B. Bojarski in the case of a Dirichlet problem. By reductio ad absurdum he proved the following result (see Theorem 4.7 in [28]).

THEOREM 0. Let $w$ be a solution to equation (3) under the above assumptions. Then $w$ can be represented in the form

$$
w(z)=f(\chi(z)) e^{\varphi(z)}+w_{0}(z) .
$$

Here $f$ is an analytic function in $\chi(D)$ while $\chi$ is a homeomorphism of the equation

$$
w_{\bar{z}}+q_{0}(z) w_{z}=0, \quad q_{0}(z):= \begin{cases}q_{1}(z)+q_{2}(z) \overline{w_{z}(z)} & \text { if } w_{z}(z) \neq 0, \\ q_{1}(z)+q_{2}(z) & \text { if } w_{z}(z)=0,\end{cases}
$$

mapping the $z$-plane onto the $\chi$-plane, $\varphi$ is a continuous function on the plane, holomorphic outside the domain $D$ and vanishing at infinity, $\chi, \varphi \in W_{p}^{1}(\mathbb{C})$, $2<p$. Moreover, $\left\|\varphi_{z}\right\|_{p},\left\|\varphi_{\bar{z}}\right\|_{p},\left\|\chi_{z}\right\|_{p},\left\|\chi_{\bar{z}}\right\|_{p}$ are bounded by a constant depending only on the domain $D$, on $q_{0}$ and $\|a\|_{p}$, $\|b\|_{p}$. In particular, $\chi$ and $\varphi$ are Hölder continuous and the Hölder exponent depends only on the same quantities. The function $w_{0} \in W_{p}^{1}(\mathbb{C})$ is a particular solution of the inhomogeneous equation (3), which is Hölder continuous on the entire plane $\mathbb{C}$, analytic outside $D$ and asymptotically 1 at infinity.

In the special case of the Beltrami equation (3) with $q_{2}=a=b=c=0$ this result is contained already in [25]. Later V. S. Vinogradov [52, 53] applied Bojarski's procedure to treat the general case of a Riemann-Hilbert boundary condition (see also Bojarski's thesis [27]) in both cases of nonnegative as well as negative index. In [54] he extended the result to quasilinear equations, which were extensively studied by Bojarski [30]. Bojarski [32] and his student Iwaniec [33, 46] considered nonlinear first order equations, too. While Bojarski and Vinogradov gave an indirect proof of the a priori estimate later Wendland $[57,58]$ and Begehr and Hsiao [13, 16] developed a direct one. This method was at the same time used in the case of the problem of linear conjugacy (see Begehr and Hile $[11,12]$ ) and for the Poincaré problem (see Begehr and Wen [18]).

There are estimates for classical as well as for weak solutions. The latter are more important because they serve to solve nonlinear problems under more general conditions.

TheOrem 1. Let D be a bounded domain the boundary of which has a Hölder 
continuously varying tangent, let $a, b \in C^{\alpha}(\bar{D})$ with

$$
\|a\|_{\alpha}+\|b\|_{\alpha} \leq K
$$

and $q_{1}, q_{2} \in C^{1+\alpha}(\bar{D})$ with

$$
\left\|q_{1}\right\|_{0}+\left\|q_{2}\right\|_{0} \leq q_{0}<1, \quad\left\|q_{1 z}\right\|_{\alpha}+\left\|q_{1 \bar{z}}\right\|_{\alpha}+\left\|q_{2 z}\right\|_{\alpha}+\left\|q_{2 \bar{z}}\right\|_{\alpha} \leq M .
$$

Then there exist constants $\gamma_{\nu}(1 \leq \nu \leq 4)$ depending on $D, z_{k}(1 \leq k \leq n), \lambda$, $\alpha, q, K$ and $M$ but not on $q_{1}, q_{2}, a, b, \varphi, \kappa, a_{k}(1 \leq k \leq n)$ such that for any $w \in C^{1+\alpha}(\bar{D})$ satisfying (7), (9) with $\log \lambda, \varphi \in C^{1+\alpha}(\partial D)$ the estimate

$$
\begin{aligned}
\|w\|_{1+\alpha} \leq & \gamma_{1}\|\varphi\|_{1+\alpha}+\gamma_{2}|\kappa|+\gamma_{3} \sum_{k=1}^{n}\left|a_{k}\right| \\
& +\gamma_{4}\left\|w_{\bar{z}}-q_{1} w_{z}-q_{2} \overline{w_{z}}-a w-b \bar{w}\right\|_{\alpha}
\end{aligned}
$$

holds. In the case of (10) instead of (9) the two middle terms are replaced by $\gamma_{2} \sum_{k=0}^{2 n}\left|b_{k}\right|$. For $n<0$ the estimate (12) is also valid, but then these middle terms do not occur.

For a direct proof see e.g. $[15,17,19,59]$. In connection with weak solutions a direct proof of the corresponding a priori estimate requires restrictive conditions on the constant $q_{0}$, i.e. on the ellipticity of (3) (see [16]). But the estimate holds without this restriction (compare $[4,30,56]$ ).

TheOREM 2. Let $D$ be a bounded domain with continuously differentiable boundary, let $a, b \in L_{p}(\bar{D})$ with

$$
\|a\|_{p}+\|b\|_{p} \leq K \quad(2<p)
$$

and let $q_{1}, q_{2}$ be measurable and satisfying (5). Then there exist constants $\gamma_{\nu}$ $(1 \leq \nu \leq 4)$ depending on $\alpha, p, q_{0}, \lambda, z_{k}(1 \leq k \leq n), K$ but not on $q_{1}, q_{2}, a$, $b, \varphi, \kappa, a_{k}(1 \leq k \leq n)$ such that for any $w \in W_{p}^{1}(\bar{D})$ satisfying $(7)$, (9) with $\log \lambda, \varphi \in C^{\alpha}(\partial D), 1<2 \alpha<2$, the estimate

$$
\begin{aligned}
\|w\|_{0} & +\left\|w_{z}\right\|_{p}+\left\|w_{\bar{z}}\right\|_{p} \\
& \leq \gamma_{1}\|\varphi\|_{\alpha}+\gamma_{2}|\kappa|+\gamma_{3} \sum_{k=1}^{n}\left|a_{k}\right|+\gamma_{4}\left\|w_{\bar{z}}-q_{1} w_{z}-q_{2} \overline{w_{z}}-a w-b \bar{w}\right\|_{p}
\end{aligned}
$$

holds.

Here the same remarks as for Theorem 1 with respect to (9) and to negative index apply. The proof for negative $n$ which is not included in $[4,15,17]$ may be given similarly to $[19,56]$.

Replacing indirect proofs of a priori estimates by direct ones is no hairsplitting because a constructive proof gives some information on the size of the constants $\gamma_{\nu}$, while an indirect proof only shows their existence. But for numerical procedures knowledge of the magnitude of the constants is important. Existence and uniqueness results for Riemann-Hilbert problems for quasilinear equations of type 
(3) and nonlinear equations are for example given in $[3,13-16,30,32,33,46,49$, 54, 57-59].

3. Discontinuous Poincaré problem for generalized Beltrami systems. The difference between the Poincaré boundary condition and the RiemannHilbert problem is that in (7), $w$ is replaced by $w_{z}$, i.e.

$$
\operatorname{Re}\left\{\overline{\lambda(z)} w_{z}(z)\right\}=\varphi(z), \quad z \in \partial D .
$$

Instead of assuming Hölder continuity here $\lambda$ and $\varphi$ are allowed to have discontinuities of first kind. While for analytic functions there is no difference in principle between (7) and (14) the discontinuous Riemann-Hilbert problem needs some extra treatment (see e.g. $[18,19,47,48,56]$ ). Problem $(3)$, (14) is reducible to this problem by setting

$$
U:=w_{z}
$$

so that after some calculations by differentiating (3)

$$
U_{\bar{z}}+\mu_{1} U_{z}+\mu_{2} \overline{U_{z}}+A U+B \bar{U}+H=0
$$

with

$$
H=\nu_{1} w_{\bar{z}}+\nu_{2} \overline{w_{\bar{z}}}+C w+D \bar{w}+E
$$

and proper coefficients (see [19], p. 312), in particular,

$$
\left|\mu_{1}(z)\right|+\left|\mu_{2}(z)\right| \leq q_{0}<1
$$

with $q_{0}$ from (5). Condition (14) means the Riemann-Hilbert condition for $U$. The connection between $w$ and $U$ is given by

$$
w(z)=w\left(z_{0}\right)+\int_{z_{0}}^{z}\left\{U(\zeta) d \zeta-\left[q_{1} U+q_{2} \bar{U}+a w+b \bar{w}+c\right](\zeta) d \bar{\zeta}\right\} .
$$

The integral involved is path-independent because of (3) and the fact that $D$ is simply connected.

The coefficients $\lambda$ and $\varphi$ in (14) are assumed to have at most a finite number of discontinuities on $\partial D$. Let $c_{1}, \ldots, c_{m}$ be those points ordered in accordance with the orientation on $\partial D$ and denote the open arcs between them by $\Gamma_{\mu}, 1 \leq \mu \leq m$, so that $\partial D \backslash\left\{c_{1}, \ldots, c_{m}\right\}=\bigcup_{\mu=1}^{m} \Gamma_{\mu}$. Then

$$
\begin{gathered}
\lambda, \varphi_{0} \in C^{\beta}\left(\overline{\Gamma_{\mu}}\right), \quad 1 \leq \mu \leq m, \quad 0<\beta<1, \quad|\lambda|=1, \\
\lambda\left(c_{\mu}-0\right)=e^{i \theta_{\mu}} \lambda\left(c_{\mu}+0\right), \quad \varphi_{\mu}:=\theta_{\mu} / \pi-k_{\mu}, \\
k_{\mu}:=\left[\theta_{\mu} / \pi\right]+I_{\mu},
\end{gathered}
$$

where $I_{\mu} \in\{0,1\}$ such that $\left|\varphi_{\mu}\right|<1$,

$$
\varphi(z)=\varphi_{0}(z) \prod_{\mu=1}^{m}\left|z-c_{\mu}\right|^{-\beta_{\mu}}, \quad 0 \leq \beta_{\mu}, \quad \beta_{\mu}+\varphi_{\mu}<1 .
$$


For this discontinuous problem

$$
\kappa:=\frac{1}{2} \sum_{\mu=1}^{m} k_{\mu}
$$

is called the index. If the set of discontinuities is empty $\kappa=n$ is an integer. But in general only $2 \kappa$ is an integer. For vanishing or positive $\kappa$ the side conditions (9) or (10) with $\kappa$ instead of $n$ serve to determine the solution of (3), (14) uniquely if, moreover, at some $z_{0} \in \bar{D}$ the value $w\left(z_{0}\right)$ is fixed. But in order to solve (16) with $U$ known for $w$ some smallness assumptions on the coefficients $a$ and $b$ have to be imposed [18, 19]. Modification of (14) for negative $\kappa$ in order to handle the conditions for the problem to be solvable again is done by replacing $\varphi$ by $\varphi+h$ where in case $D=\mathbb{D}$

$$
h(z):= \begin{cases}\sum_{k=\kappa+1}^{-\kappa-1} h_{k} z^{k}, h_{-k}=\overline{h_{k}}(|k| \leq-\kappa-1) & \text { if }-\kappa \in \mathbb{N}, \\ \sum_{k=[\kappa]+2}^{-[\kappa]} h_{k} z^{k-1 / 2}, h_{1-k}=\overline{h_{k}}(|k| \leq[-\kappa]) & \text { if } \frac{1}{2}-\kappa \in \mathbb{N},\end{cases}
$$

with unknown coefficients $h_{k}$ to be determined properly. As before this function has to be modified for general $D$. Using Muskhelishvili's theory of singular integrals in [19] via direct estimation rather than by reductio ad absurdum an a priori estimate for solutions to the modified problem $(3),(14),(10)$ (with $n=\kappa$ ) is proved.

THEOREM 3. Let $D$ be a bounded domain with continuously differentiable boundary, let

$$
\begin{gathered}
\left|q_{1}(z)\right|+\left|q_{2}(z)\right| \leq q_{0}<1, \quad\left\|q_{1 z}\right\|_{p}+\left\|q_{2 z}\right\|_{p} \leq K \quad(2<p), \\
|a(z)|+|b(z)| \leq \varepsilon K, \quad\left\|a_{z}\right\|_{p}+\left\|b_{z}\right\|_{p} \leq \varepsilon K, \quad 2 \varepsilon K<1,
\end{gathered}
$$

and suppose $\lambda$ and $\varphi$ have the properties described above. Then under some more technical restrictions there exist nonnegative constants $\gamma_{\mu}$ depending on $\alpha, \varphi_{\mu}, \beta_{\mu}$ $(1 \leq \mu \leq m)$, and $M$ depending on $p, q_{0}, \alpha, \beta, \lambda, \varepsilon, K, c_{\mu}, \varphi_{\mu}, \beta_{\mu}(1 \leq \mu \leq m)$, $z_{k}(0 \leq k \leq 2 n)$ but not on $w, q_{1}, q_{2}, a, b, c, \varphi, b_{k}(0 \leq k \leq 2 n)$ such that

$$
\begin{aligned}
\|w\|_{\alpha}+\left\|\prod_{\mu=1}^{m}\left(z-c_{\mu}\right)^{\gamma_{\mu}} w_{z}\right\|_{0}+ & \left\|\prod_{\mu=1}^{m}\left(z-c_{\mu}\right)^{\gamma_{\mu}} w_{\bar{z}}\right\|_{0} \\
& \leq M\left\{\left\|\varphi_{0}\right\|_{\beta}+\sum_{k=0}^{2 \kappa}\left|b_{k}\right|+\|c\|_{0}+\left\|c_{z}\right\|_{p}\right\}
\end{aligned}
$$

for any solution to (3), (14), (10) (with $w_{z}$ instead of $w$ ). Here for negative index $\kappa$ neither the side condition (10) for $w_{z}$ nor the second term on the right hand side of (18) occur.

Related nonlinear problems are discussed in [18, 19]. 
4. Riemann problem for generalized Beltrami systems. The main problem in solving the linear Riemann problem for (3) is to find proper entire solutions to (3), i.e. functions satisfying (3) in the whole plane. The reason is that proper analytic functions can be used to reduce the general problem to an equation (3) without any jump condition on $\Gamma$ (see e.g. [10, 11, 39]). For, say, a simple, smooth arc $\Gamma$ in $\mathbb{C}$ and a function $\Phi$ defined on both sides of $\Gamma$ define

$$
\Phi^{ \pm}(\zeta):=\lim _{z \rightarrow \zeta} \Phi(z),
$$

where $z$ not on $\Gamma$ nontangentially tends to a point $\zeta$ of $\Gamma$ different from the endpoints, approaching $\Gamma$ from the right and from the left hand side, respectively.

Let, for simplicity, $\Gamma$ be a single, simply closed, smooth curve in $\mathbb{C}$ rather than a finite set of such curves, mutually disjoint, and let $G$ and $g$ be Hölder continuous functions defined on $\Gamma$ such that $|G(z)|=1$ on $\Gamma$. Denote by $D^{+}$and $D^{-}$the inner and outer domain of $\Gamma$, respectively. We look for a solution to (3) in $\mathbb{C} \backslash \Gamma$ satisfying

$$
w^{+}=G w^{-}+g \quad \text { on } \Gamma
$$

and e.g. vanishing at infinity. Let $X$ be the analytic factorization of $G$, i.e. the analytic function behaving as $z^{-n}$ at infinity and satisfying the homogeneous condition (19):

$$
X^{+}=G X^{-} \text {. }
$$

This function is given by

where

$$
X(z):= \begin{cases}\exp L(z), & z \in D^{+}, \\ z^{-n} \exp L(z), & z \in D^{-}\end{cases}
$$

$$
n=\operatorname{ind} G=\frac{1}{2 \pi i} \int_{\Gamma} d \log G(\zeta) \in \mathbb{Z}
$$

is the index of (19). Moreover, let

$$
\varphi(z):=\frac{1}{2 \pi i} \int_{\Gamma} \frac{g(\zeta)}{X^{+}(\zeta)} \frac{d \zeta}{\zeta-z} \quad(z \notin \Gamma) .
$$

This function is analytic and as the Plemelj-Sokhotskiı formula shows (see [39, $45,48]$ ), it satisfies

$$
\varphi^{+}=\varphi^{-}+\frac{g}{X^{+}} \quad \text { on } \Gamma \text {. }
$$

Then, obviously,

$$
\left(\frac{w}{X}-\varphi\right)^{+}=\left(\frac{w}{X}-\varphi\right)^{-} \text {on } \Gamma .
$$

Hence, $\omega:=w / X-\varphi$ is continuous on $\Gamma$ and satisfies in $\mathbb{C} \backslash \Gamma$

$$
\omega_{\bar{z}}+q_{1} \omega_{z}+q_{2} \overline{\omega_{z}}+\left(a+\frac{X^{\prime}}{X} q_{1}\right) \omega+\left(b \frac{\bar{X}}{X}+\frac{\overline{X^{\prime}}}{X} q_{2}\right) \bar{\omega}+\widetilde{c}=0
$$




$$
\widetilde{c}:=\frac{c}{X}+q_{1} \varphi^{\prime}+q_{2} \overline{\varphi^{\prime}}+\left(a+\frac{X^{\prime}}{X} q_{1}\right) \varphi+\left(b \frac{\bar{X}}{X}+\frac{\overline{X^{\prime}}}{X} q_{2}\right) \bar{\varphi} .
$$

The behaviour of $\omega$ at infinity is controlled by that of $w$ and $X$.

In order to prove an a priori estimate for entire solutions to (3) this equation is stepwise reduced first to an inhomogeneous, then to a homogeneous Beltrami equation and finally to the Cauchy-Riemann equation (see [10, 11]). A proper space for solutions is the space of functions with derivatives in

$$
L_{\left(p, p^{\prime}\right)}:=L_{p} \cap L_{p^{\prime}}, \quad 1 \leq p^{\prime}<2<p<\infty,
$$

and in $L_{p, 2}$ for $2<p<\infty$, respectively (see [51]).

Theorem 4. Besides (5), let for some $\varepsilon, 0<\varepsilon<1$,

$$
\left|q_{1}(z)\right|+\left|q_{2}(z)\right|=O\left(|z|^{-\varepsilon}\right) \quad \text { as } z \rightarrow \infty,
$$

and let $a, b \in L_{\left(p, p^{\prime}\right)} \cap L_{p, 2}$ for suitable $p, p^{\prime}$ satisfying in particular

$$
\frac{2}{1+\varepsilon}<p^{\prime}<2<p<\frac{4}{2-\varepsilon},
$$

with

$$
\|a\|_{\left(p, p^{\prime}\right)}+\|b\|_{\left(p, p^{\prime}\right)} \leq K .
$$

Let $w$ be an entire function vanishing at infinity and such that $w_{z}, w_{\bar{z}} \in L_{\left(p, p^{\prime}\right)}$, $w_{\bar{z}} \in L_{p, 2}$. Then there exists a constant $M$ depending on $K, \varepsilon, p, p^{\prime}$ and $q_{0}$ such that

(20) $\|w\|_{0}+\left\|w_{z}\right\|_{\left(p, p^{\prime}\right)}+\left\|w_{\bar{z}}\right\|_{\left(p, p^{\prime}\right)} \leq M\left\|w_{\bar{z}}+q_{1} w_{z}+q_{2} \overline{w_{z}}+a w+b \bar{w}\right\|_{\left(p, p^{\prime}\right)}$.

Moreover, equation (3) under the above conditions together with $c \in L_{\left(p, p^{\prime}\right)} \cap$ $L_{p, 2}$ can be shown to be uniquely solvable by an entire function satisfying (20). In particular, (20) shows that the homogeneous problem, i.e. $c \equiv 0$, is only trivially solvable (see [11]). If instead of being an entire solution $w$ satisfies

$$
w^{+}=w^{-}+g \quad \text { on } \Gamma
$$

with $g \in C^{\alpha}(\Gamma)$ then (20) has to be replaced by

$$
\begin{aligned}
\|w\|_{0}+\left\|w_{z}\right\|_{\left(p, p^{\prime}\right)} & +\left\|w_{\bar{z}}\right\|_{\left(p, p^{\prime}\right)} \\
& \leq M\left\{\|g\|_{\alpha}+\left\|w_{\bar{z}}+q_{1} w_{z}+q_{2} \overline{w_{z}}+a w+b \bar{w}\right\|_{\left(p, p^{\prime}\right)}\right\} .
\end{aligned}
$$

While here solutions in the weak sense are involved, classical ones may be considered, too. The disadvantage then is that the $\Pi$-operator (see [51], Chapter I, $\S 8)$

$$
(\Pi f)(z):=-\frac{1}{\pi} \int_{\mathbb{C}} f(\zeta) \frac{d \xi d \eta}{(\zeta-z)^{2}} \quad(\zeta=\xi+i \eta)
$$

for $f \in C^{\alpha}(\mathbb{C}) \cap L_{p}(\mathbb{C})$ may be estimated by

$$
\|\Pi f\|_{p, \alpha} \leq M(p, \alpha)\|f\|_{p, \alpha}
$$


where $\|f\|_{p, \alpha}:=\|f\|_{p}+\|f\|_{\alpha}$ and $M(p, \alpha)$ is some fixed constant $>1$, while for $f \in L_{p}$

$$
\|\Pi f\|_{p} \leq \Lambda_{p}\|f\|_{p}
$$

with $\Lambda_{p}$ continuous in $p$ and $\Lambda_{2}=1$. Hence, an estimate of type (20) for classical solutions to (3) in $\mathbb{C}$ can be proven only under strong restrictions, namely $q_{0}$ has to be so small that $M(p, \alpha) q_{0}<1$. In order to get a better result, the constant $M(p, \alpha)$ has to be considered in more detail. In [10] for the case $q_{1}=q_{2}=0$ the following result is derived.

Theorem 5. For some $\varepsilon, 0<\varepsilon<1$, let

$$
\left(1+|z|^{1+\varepsilon}\right)[|a(z)|+|b(z)|] \leq K
$$

and let $g \in C^{\alpha}(\Gamma)$ for $\alpha=(p-2) / p$, where $2<p<2 /(1-\varepsilon)$. Then there exists a constant $M$ depending on $K, \varepsilon, p$ and $\Gamma$ such that for any function $w$ with $w_{\bar{z}} \in L_{p, 2}(\mathbb{C})$ satisfying the jump condition $w^{+}=w^{-}+g$ on $\Gamma$ and vanishing at infinity we have

$$
\left\|w^{ \pm}\right\|_{\alpha} \leq M\left[\|g\|_{\alpha}+\left\|w_{\bar{z}}+a w+b \bar{w}\right\|_{p, 2}\right],
$$

where $\left\|w^{ \pm}\right\|_{\alpha}:=\|w\|_{\alpha, \overline{D^{+}}}+\|w\|_{\alpha, \overline{D^{-}}}$.

5. Second order elliptic equations. A priori estimates for solutions to the Dirichlet problem for elliptic equations of second order

$$
L u:=\sum_{j, k=1}^{m} a_{j k} u_{x_{j} x_{k}}+\sum_{j=1}^{m} b_{j} u_{x_{j}}+c u=d
$$

in a regular domain $D \in C^{2, \alpha}$ of $\mathbb{R}^{m}$ follow from Schauder estimates (see e.g. [40], $6.2)$. Let $a_{j k}, b_{j}, c, d \in C^{\alpha}(\bar{D}),\left(a_{j k}\right)$ being symmetric and satisfying

$$
\lambda \sum_{j=1}^{m} \xi_{j}^{2} \leq \sum_{j, k=1}^{m} a_{j k} \xi_{j} \xi_{k}
$$

for some positive constant $\lambda$ for all real $\xi=\left(\xi_{1}, \ldots, \xi_{m}\right)$ in $D$. Moreover, suppose

$$
\|a\|_{\alpha}:=\sum_{j, k=1}^{m}\left\|a_{j k}\right\|_{\alpha},\|b\|_{\alpha}:=\sum_{j=1}^{m}\left\|b_{j}\right\|_{\alpha},\|c\|_{\alpha} \leq \Lambda .
$$

Then there exists a constant $C$ depending on $m, \alpha, \lambda, \Lambda$ and $D$ such that

$$
\|u\|_{2, \alpha, D} \leq C\left\{\|u\|_{0, D}+\|u\|_{2, \alpha, \partial D}+\|L u\|_{\alpha, D}\right\}
$$

(see e.g. [60]). If, moreover, $c \leq 0$ on $D$ the maximum principle is valid, giving

$$
\|u\|_{0, D} \leq K\left\{\|u\|_{0, \partial D}+\|L u\|_{0, D}\right\}
$$

for any $u \in C^{2, \alpha}(\bar{D})$, where $K$ depends on $\lambda, \Lambda$ and $D$. Hence, the following result holds (see [1]). 
THEOREM 6. Under the above assumptions there exists a constant $M$ depending on $m, \alpha, \lambda, \Lambda$ and $D$ such that for all $u \in C^{2, \alpha}(\bar{D})$

$$
\|u\|_{2, \alpha, D} \leq M\left\{\|u\|_{2, \alpha, \partial D}+\|L u\|_{\alpha, D}\right\} .
$$

As in the case of the generalized Beltrami equation the Dirichlet condition has to be replaced by some growth condition if instead of a bounded domain the elliptic equation is considered in the whole space $\mathbb{R}^{m}$. Because an elliptic equation satisfying (24) locally may be reduced to the canonical form it is no restriction to assume $L$ to behave asymptotically as the Laplace operator $\Delta$ at infinity. More precisely, in $[12]$ it is assumed that for some positive $\varepsilon$

$$
\begin{gathered}
a_{j k}(x)-\delta_{j k}=O\left(|x|^{-\varepsilon}\right), \quad b_{j}(x)=O\left(|x|^{-1-\varepsilon}\right), \\
c(x)=O\left(|x|^{-2-\varepsilon}\right), \quad d(x)=O\left(|x|^{-2-\varepsilon}\right) \quad \text { as }|x| \rightarrow \infty .
\end{gathered}
$$

A proper kind of Hölder norm in the space of certain entire functions satisfying some growth condition is

$$
\|u\|_{\sigma, \alpha}:=\|u\|_{\sigma}+\|u\|_{(\sigma, \alpha)}
$$

where

$$
\begin{gathered}
\|u\|_{\sigma}:=\sup _{x \in \mathbb{R}^{m}}(1+|x|)^{-\sigma}|u(x)|, \\
\|u\|_{(\sigma, \alpha)}:=\sup _{\substack{x, y \in \mathbb{R}^{m} \\
0<2|x-y| \leq 1+|x|}}(1+|x|)^{\alpha-\sigma} \frac{|u(x)-u(y)|}{|x-y|^{\alpha}}
\end{gathered}
$$

with some real $\sigma$ and $0<\alpha<1$. Let $\nabla$ denote the gradient operator and $\nabla_{2}=$ $\left(\partial^{2} / \partial x_{j} \partial x_{k}\right)$ the Hesse matrix rather than the Laplacian, $a=\left(a_{j k}\right), b=\left(b_{j}\right)$. Similarly to the case of a bounded domain, a Schauder estimate can be derived (see $[12])$.

Theorem 7. Let $u \in C^{2}\left(\mathbb{R}^{m}\right), 2 \leq m$, with $\|u\|_{\sigma}$ finite for some real $\sigma$, let (24) hold in $\mathbb{R}^{m}$ and

$$
\|a\|_{0, \alpha},\|b\|_{-1, \alpha},\|c\|_{-2, \alpha} \leq \Lambda .
$$

Then there exists a constant $C$ depending on $m, \alpha, \sigma, \lambda$ and $\Lambda$ but not on $u, a$, $b, c$ such that

$$
\|u\|_{\sigma, 1}+\|\nabla u\|_{\sigma-1,1}+\left\|\nabla_{2} u\right\|_{\sigma-2, \alpha} \leq C\left\{\|u\|_{0}+\|L u\|_{\sigma-2, \alpha}\right\} .
$$

This estimate leads to a priori estimates. But there is a crucial difference between the cases $m=2$ and $2<m$. While for the latter the homogeneous equation

$$
\Delta u+c u=0
$$

under the above decay condition on $c$ at infinity only has the trivial solution in $\mathbb{R}^{m}$ vanishing at infinity, the situation for $m=2$ is more involved. In that case, as for the Poisson equation, an entire solution in general grows as a multiple of 
$\log |x|$. But solutions of this kind need not be unique, as is shown in [12] by an example. Hence, for $m=2$ in the sequel it is assumed that $c \equiv 0$.

THEOREM 8. Suppose there exist constants $0<\delta, 0<\alpha<1,0 \leq \Lambda$ such that

$$
\|a-I\|_{-\delta, \alpha},\|b\|_{-1-\delta, \alpha},\|c\|_{-2-\delta, \alpha} \leq \Lambda,
$$

where $I=\left(\delta_{j k}\right)$ denotes the $m \times m$ identity matrix. Let $c \leq 0$ and $c \equiv 0$ if $m=2$ and $u \in C^{2}\left(\mathbb{R}^{m}\right)$.

(a) If for $3 \leq m$ and $2<\tau<m$ the function $u$ has finite norm $\|u\|_{2-\tau}$, then

$$
\|u\|_{2-\tau, 1}+\|\nabla u\|_{1-\tau, 1}+\left\|\nabla_{2} u\right\|_{-\tau, \alpha} \leq M\|L u\|_{-\tau, \alpha},
$$

where $M=M(m, \alpha, \delta, \tau, \lambda, \Lambda)$.

(b) For $m=2$ assume that $u(x)-\gamma \log |x|$ vanishes at infinity for some constant $\gamma$. Then for any $\varepsilon>0$

$$
\|u\|_{\varepsilon, 1}+\|\nabla u\|_{\varepsilon-1,1}+\left\|\nabla_{2} u\right\|_{\varepsilon-2, \alpha} \leq M\|L u\|_{-\tau, \alpha},
$$

and also $|\gamma| \leq M\|L u\|_{-\tau, \alpha}$, where $M=M(\alpha, \delta, \tau, \lambda, \Lambda)$. If, moreover, $0<\varepsilon<1$, $\varepsilon \leq \delta, 2+\varepsilon \leq \tau$, then

$$
\|\Delta u\|_{-2-\varepsilon, \alpha} \leq M\|L u\|_{-\tau, \alpha}
$$

and for $1 \leq|x|$

$$
|u(x)-\gamma \log | x|| \leq M\|L u\|_{-\tau, \alpha}(1+|x|)^{-\varepsilon} \log (1+|x|)
$$

with $M=M(\alpha, \delta, \tau, \varepsilon, \lambda, \Lambda)$.

This result in [12] is used to give an existence and uniqueness proof for entire solutions not only with the indicated behaviour at infinity but also for solutions which behave asymptotically as a harmonic polynomial to which in case $m=2$ a term of the kind $\gamma \log |x|$ is added. These solutions are shown to exist under some stronger decay conditions on the coefficients than those formulated in Theorem 8 . In order to study nonlinear equations the Hölder norms are not suitable as was mentioned earlier but proper $L_{p}$-estimates are not yet available.

6. Initial and boundary value problem for a composite type system. The simplest form of a general linear composite system of first order of three real equations in complex form is

$$
\begin{gathered}
w_{\bar{z}}+q_{1} w_{z}+q_{2} \overline{w_{z}}+a_{1} w+a_{2} \bar{w}+a_{3} \omega+c=0, \\
\omega_{y}+b_{1} w+b_{2} \bar{w}+b_{3} \omega+d=0,
\end{gathered}
$$

where the coefficients are complex except $a_{3}, b_{3}$ and $d$ and such that $b_{2}=\overline{b_{1}}$. The unknown function $w$ is complex while $\omega$ is real.

Any second order elliptic equation in the plane can be reduced to such a system. For simplicity (31) is studied in the unit disc $\mathbb{D}$ of the complex plane $\mathbb{C}$. Natural initial and boundary conditions are

$$
\operatorname{Re}\{\bar{\lambda} w\}=\varphi \quad \text { on } \Gamma=\partial \mathbb{D}, \quad \omega=\psi \quad \text { on } \gamma=\{|z|=1, \operatorname{Im} z \leq 0\}
$$


where $|\lambda(z)|=1, \varphi$ and $\psi$ are real-valued. As in Section 2 the Riemann-Hilbert problem (7) is influenced by its index $n$, so is the case for problem (32). Hence for $n \geq 0$ the side conditions (10) are imposed in order to get unique solutions of problem (31), (32) while for its solvability for $n<0$ the first condition in (32) is modified by replacing $\varphi$ by $\varphi+h$ with $h$ from (11).

TheOREm 9. Let $a_{3}=b_{1}=b_{2}=0$, let $q_{1}, q_{2}$ satisfy (5), suppose that

$$
\left\|a_{1}\right\|_{p}+\left\|a_{2}\right\|_{p} \leq K \quad(2<p), \quad\left\|b_{3}\right\|_{\alpha^{\prime}} \leq K \quad\left(0<\alpha^{\prime}<1\right),
$$

and let $\lambda, \varphi \in C^{\alpha}(\Gamma)(1 / 2<\alpha<1), \psi \in C^{\alpha^{\prime}}(\gamma), c \in L_{p}(\overline{\mathbb{D}})$ and $d \in C^{\alpha^{\prime}}(\overline{\mathbb{D}})$. Then any solution $(w, \omega)$ of (31), (32) satisfies $w \in W_{p_{0}}^{1}(\mathbb{D}), 2<p_{0} \leq \min \{p$, $1 /(1-\alpha)\}, \omega \in C_{y}^{1}(\overline{\mathbb{D}})$ and

$$
\begin{gathered}
\|w\|_{\beta}+\left\|w_{z}\right\|_{p_{0}}+\left\|w_{\bar{z}}\right\|_{p_{0}} \leq M_{1}\left\{\|\varphi\|_{\alpha}+\sum_{\mu=0}^{2 n}\left|b_{\mu}\right|+\|c\|_{p}\right\}, \\
\|\omega\|_{\beta}+\left\|\omega_{y}\right\|_{0} \leq M_{2}\left\{\|\psi\|_{\alpha^{\prime}}+\|d\|_{\alpha^{\prime}}\right\}
\end{gathered}
$$

where $0<\beta \leq \min \left\{\alpha, \alpha^{\prime}, 1-2 / p_{0}\right\}$ and $M_{1}$ and $M_{2}$ depend on $\alpha, p, p_{0}, q_{0}, K$, $\lambda$ and on $\alpha^{\prime}$ and $K$, respectively.

THEOREM 10. Let the coefficients of problem (31), (32) satisfy the conditions of the preceding theorem but $a_{3} \in L_{p}(\overline{\mathbb{D}}), b_{1}, b_{2} \in C^{\beta}(\overline{\mathbb{D}}), b_{2}=\overline{b_{1}}$, with

$$
2 M_{1} M_{2}\left\|a_{3}\right\|_{p}\left\|b_{1}\right\|_{\beta} \leq k<1 .
$$

Then any solution of (31), (32) satisfies

$$
\begin{aligned}
\|w\|_{\beta}+\left\|w_{z}\right\|_{p_{0}}+\left\|w_{\bar{z}}\right\|_{p_{0}} & +\|\omega\|_{\beta}+\left\|\omega_{y}\right\|_{0} \\
& \leq M\left\{\|\varphi\|_{\alpha}+\|\psi\|_{\alpha^{\prime}}+\sum_{\mu=0}^{2 n}\left|b_{\mu}\right|+\|c\|_{p}+\|d\|_{\alpha^{\prime}}\right\},
\end{aligned}
$$

where $M$ depends on $\alpha, \alpha^{\prime}, \beta, p, p_{0}, q_{0}, k, K$ and $\lambda$.

In both estimates (33) and (34) the term $\sum_{\mu=0}^{2 n}\left|b_{\mu}\right|$ has to be replaced by 0 if $n$ is negative. These results are proved in [20].

7. Initial and boundary value problem for a pseudoparabolic equation. A pseudoparabolic equation related to the generalized Beltrami equation (3) is

$$
\begin{aligned}
w_{t \bar{z}}+q_{1} w_{t z}+q_{2} \overline{w_{t z}}+ & a_{1} w_{t}+a_{2} \overline{w_{t}} \\
& +\widehat{b}_{1} w_{\bar{z}}+\widehat{b}_{2} \overline{w_{\bar{z}}}+\widehat{b}_{3} w_{z}+\widehat{b}_{4} \overline{w_{z}}+c_{1} w+c_{2} \bar{w}+f=0 .
\end{aligned}
$$

Here the coefficients are functions of $(t, z) \in I \times D$ where $I=[0, T]$ is a compact interval with some positive $T$ and $D$ is a simply connected domain in $\mathbb{C}$ with smooth boundary $\partial D$. 
For any Banach space $V$ the spaces of continuous and of continuously differentiable mappings, respectively, from $I$ into $V$ endowed with the norms

$$
\|w\|_{0, V}:=\sup _{t \in I}\|w(t)\|_{V}, \quad\|w\|_{1, V}:=\|w\|_{0, V}+\left\|w^{\prime}\right\|_{0, V}
$$

are denoted by $C(I ; V)$ and $C^{1}(I ; V)$, respectively. They are Banach spaces themselves. Quite obviously Theorem 2 can be generalized to (see $[5,35]$ )

Theorem 11. Let $D$ be a $C^{1}$ domain, and let $\alpha$ and $p$ be real numbers satisfying

$$
1<2 \alpha<2<p<\frac{1}{1-\alpha} .
$$

Let $\lambda \in C^{\alpha}(\partial D), a, b, c \in C\left(I ; L_{p}(\bar{D})\right), g \in C^{1}\left(I ; C^{\alpha}(\partial D)\right), a_{k} \in \partial D, b_{k} \in C^{1}(I)$ for $0 \leq k \leq 2 n, d \in W_{p}^{1}(D)$. Then any solution $W \in C^{1}\left(I ; W_{p}^{1}(D)\right)$ of the problem

$$
\begin{gathered}
w_{t \bar{z}}(t, z)+a(t, z) w_{t}(t, z)+b(t, z) \overline{w_{t}(t, z)}=c(t, z) \quad \text { in } I \times D, \\
\operatorname{Re}\{\overline{\lambda(z)} w(t, z)\}=g(t, z)+h(t, z) \quad \text { in } I \times \partial D, \\
\operatorname{Im}\left\{\overline{\lambda\left(a_{k}\right)} w\left(t, a_{k}\right)\right\}=b_{k}(t) \quad(0 \leq k \leq 2 n, 0 \leq n) \quad \text { in } I, \\
w(0, z)=d(z) \quad \text { in } D
\end{gathered}
$$

satisfies the a priori estimate

$$
\begin{aligned}
\|w\|_{1, W_{p}^{1}(D)} \leq & \gamma_{1}\|g\|_{1, C^{\alpha}(\partial D)}+\gamma_{2} \sum_{k=0}^{2 n}\left\|b_{k}\right\|_{C^{1}(I)} \\
& +\gamma_{3}\|c\|_{0, L_{p}(\bar{D})}+\gamma_{4}\|d\|_{W_{p}^{1}(D)},
\end{aligned}
$$

where the constants $\gamma_{1}, \ldots, \gamma_{4}$ depend on $\alpha, p, a_{k}, T, D, \lambda$ and on an upper bound for $\|a\|_{0, L_{p}(\bar{D})}+\|b\|_{0, L_{p}(\bar{D})}$. Here $h(t, z)=0$ if $0 \leq n$ and for $n<0$

$$
h(t, z):=\sum_{\nu=n+1}^{-n-1} h_{\nu}(t) \omega^{\nu}(z), \quad h_{-\nu}(t)=\overline{h_{\nu}(t)} \quad(|\nu| \leq-n-1),
$$

where $\omega$ is the conformal mapping from $D$ onto the unit disc satisfying $\omega(0)=0$, $\omega^{\prime}(0)>0$ and $h_{\nu} \in C^{1}(I)$ are to be determined properly, so that for $n<0,(35)$ is solvable. Moreover, the compatibility conditions

$$
\begin{aligned}
\operatorname{Re}\{\overline{\lambda(z)} d(z)\} & =g(0, z)+h(0, z) \quad \text { on } \partial D, \\
\operatorname{Im}\left\{\overline{\lambda\left(a_{k}\right)} d\left(a_{k}\right)\right\} & =b_{k}(0) \quad(0 \leq k \leq 2 n, 0 \leq n)
\end{aligned}
$$

are assumed to hold.

A similar a priori estimate can be obtained for the initial Riemann-Hilbert boundary problem for the more general linear pseudoparabolic equation

$$
\begin{aligned}
w_{t \bar{z}}+q_{1} w_{t z}+q_{2} \overline{w_{t z}}+a_{1} w_{t}+a_{2} \overline{w_{t}} & \\
& +\widehat{b}_{1} w_{\bar{z}}+\widehat{b}_{2} \overline{w_{\bar{z}}}+\widehat{b}_{3} w_{z}+\widehat{b}_{4} \overline{w_{z}}+c_{1} w+c_{2} \bar{w}+f=0
\end{aligned}
$$


under appropriate conditions (see $[5,35])$. In this case the constants $\gamma_{1}, \ldots, \gamma_{4}$ depend on $\left\|a_{1}\right\|_{0, L_{p}(\bar{D})}+\left\|a_{2}\right\|_{0, L_{p}(\bar{D})}, \sum_{j=1}^{4}\left\|\widehat{b}_{j}\right\|_{0, L_{\infty}(\bar{D})},\left\|c_{1}\right\|_{0, L_{p}(\bar{D})}+\left\|c_{2}\right\|_{0, L_{p}(\bar{D})}$, too. The proof of (36) is based on

LEMma. Under the assumptions of Theorem 11 but with $g \in C\left(I ; C^{\alpha}(\partial D)\right)$ any solution to the equation

$$
w_{\bar{z}}(t, z)+a(t, z) w(t, z)+b(t, z) \overline{w(t, z)}=c(t, z) \quad \text { in } I \times D
$$

satisfying the boundary and side conditions from (35) but no initial condition fulfils for any $t \in I$ the estimate

$$
\|w(t, \cdot)\|_{W_{p}^{1}(D)} \leq \gamma_{1}\|g(t, \cdot)\|_{C^{\alpha}(\partial D)}+\gamma_{2} \sum_{k=0}^{2 m}\left|b_{k}(t)\right|+\|c(t, \cdot)\|_{L_{p}(\bar{D})} .
$$

Here $\gamma_{1}, \gamma_{2}, \gamma_{3}$ depend on $t, \alpha, p, q_{0}, a_{k}, D, \lambda$ and on an upper bound for $\|a(t, \cdot)\|_{L_{p}(\bar{D})}+\|b(t, \cdot)\|_{L_{p}(\bar{D})}$.

8. Half-Dirichlet problems for first order elliptic equations on the unit ball in higher dimensions. The elements of the complex Clifford algebra $\mathbb{C}_{m}(3 \leq m)$ are represented in the form $a=\sum_{A} a_{A} e_{A}$ with $a_{A} \in \mathbb{C}, A=$ $\left\{\alpha_{1}, \ldots, \alpha_{h}\right\} \subset\{1, \ldots, m\}, 1 \leq \alpha_{1}<\ldots<\alpha_{h} \leq m$. The basis elements

$$
e_{A}=e_{\alpha_{1} \ldots \alpha_{h}}=e_{\alpha_{1}} \ldots e_{\alpha_{h}}, \quad e_{\emptyset}=e_{0}
$$

obey the multiplication rules

$$
e_{j} e_{k}+e_{k} e_{j}=-2 \delta_{j k}, \quad 1 \leq j, k \leq m .
$$

For more details see $[34,41]$. Each element $x=\left(x_{1}, \ldots, x_{m}\right) \in \mathbb{R}^{m}$ is identified with the element $x=\sum_{j=1}^{m} x_{j} e_{j}$ of $\mathbb{C}_{m}$. The Dirac operator

$$
\bar{\partial}:=\sum_{j=1}^{m} e_{j} \frac{\partial}{\partial x_{j}}
$$

is related to the Laplace operator in $\mathbb{R}^{m}$ by $\bar{\partial}^{2}=-\Delta$. Its fundamental solution is

$$
E(x)=-\frac{1}{\omega_{m}} \frac{x}{|x|^{m}},
$$

where $|x|:=\left(\sum_{j=1}^{m} x_{j}^{2}\right)^{1 / 2}$ and $\omega_{m}$ is the area of the unit sphere in $\mathbb{R}^{m}$.

Let $J_{j}$ denote the (real-) linear mappings from $\mathbb{C}_{m}$ into itself satisfying

$$
J_{j}\left(e_{j}\right)=-e_{j}, \quad J_{j}\left(e_{k}\right)=e_{k} \text { for } j \neq k \quad(1 \leq j, k \leq m) .
$$

Every real-linear mapping from $\mathbb{C}_{m}$ into itself may be written in the form

$$
\sum_{A} C_{A} J_{A}(a), \quad C_{A} \in \mathbb{C}_{m},
$$


where for $A=\left\{\alpha_{1}, \ldots, \alpha_{h}\right\}, 0 \leq \alpha_{1}<\ldots<\alpha_{h} \leq m, J_{A}:=J_{\alpha_{1}} \ldots J_{\alpha_{h}}, J_{\emptyset}$ is the identity mapping and $J_{0}$ is given by

$$
J_{0}\left(\sum_{A} a_{A} e_{A}\right)=\sum_{A} \overline{a_{A}} e_{A} .
$$

In $[36,61]$ the following special boundary value problem in the unit ball $B$ is studied. For given $F \in L_{p}\left(\bar{B} ; \mathbb{C}_{m}\right)(m<p), C_{A}(x) \in C\left(\bar{B} ; \mathbb{C}_{m}\right), f \in C^{\alpha}\left(\partial B ; \mathbb{C}_{m}\right)$ $(0<\alpha<1$ or $\alpha=(p-m) / p)$ find a $w \in C^{\alpha}\left(\bar{B} ; \mathbb{C}_{m}\right)$ such that $\bar{\partial} w \in L_{p}\left(\bar{B} ; \mathbb{C}_{m}\right)$ $(m<p)$ and

$$
\begin{gathered}
\bar{\partial} w(x)+\sum_{A} C_{A}(x) J_{A} w(x)=F(x) \quad \text { in } B, \\
(1+i x)(w(x)-f(x))=0 \quad \text { on } \partial B .
\end{gathered}
$$

Since for $|x|=1$ we have

$$
\frac{1+i x}{2} \frac{1-i x}{2}=0, \quad \frac{1+i x}{2}+\frac{1-i x}{2}=1,
$$

this boundary condition is called a half-Dirichlet condition.

While the operator $T_{D}$ given by

$$
T_{D} \phi(x):=\frac{1}{\omega_{m}} \int_{D} \frac{y-x}{|y-x|^{m}} \phi(y) d y
$$

on $L_{p}\left(\bar{D} ; \mathbb{C}_{m}\right)$ for any domain $D$ in $\mathbb{R}^{m}$ is useful for treating differential equations for the operator $\bar{\partial}$, in the case of $D=B$ the operator $T_{1}$ is more appropriate, where

$$
T_{1} \phi(x):=\frac{1}{\omega_{m}} \int_{B}\left\{\frac{y-x}{|y-x|^{m}}-i \frac{1+x y}{|1+x y|^{m}}\right\} \phi(y) d y .
$$

It obviously satisfies $(1+i x) T_{1} \phi(x)=0$ on $\partial B$. Moreover, it is a compact operator on $L_{p}\left(\bar{B} ; \mathbb{C}_{m}\right)$ satisfying $\bar{\partial} T_{1} \phi(x)=\phi(x)$ in $B$. Hence, by means of the operator $T_{1}$ problem (38) can be reduced to the half-Dirichlet problem for left regular functions in $B$. The solution to this problem is given by

$$
K f(x):=-\frac{1}{\omega_{n}} \int_{B} \frac{y-x}{|y-x|^{m}} d \sigma_{y}(1+i y) f(y),
$$

where the surface element $d \sigma_{y}$ is

$$
d \sigma_{y}=\sum_{j=1}^{m}(-1)^{j-1} e_{j} d y_{1} \wedge \ldots \wedge d y_{j-1} \wedge d y_{j+1} \wedge \ldots \wedge d y_{m}
$$

(see [41]). Problem (38) is then seen to be equivalent to the integral equation

$$
v(x)+\sum_{A} C_{A}(x) J_{A}\left(T_{1} v(x)\right)=F(x)+\sum_{A} C_{A}(x) J_{A}(K f(x)) .
$$


The relation of $w$ and $v$ is

$$
w(x)=T_{1} v(x)+K f(x) .
$$

For $C_{A} \in C\left(\bar{B} ; \mathbb{C}_{m}\right), A \subset\{0,1, \ldots, m\}$ the operator $\sum_{A} C_{A} J_{A}\left(T_{1}\right)$ is a bounded linear operator on $L_{p}\left(\bar{B} ; \mathbb{C}_{m}\right)$ satisfying

$$
\left\|\sum_{A} C_{A} J_{A}\left(T_{1} v\right)\right\|_{p} \leq C\left\|\sum_{A} C_{A}\right\|_{0}\|v\|_{p} .
$$

Here $C$ is a suitable real constant independent of the $C_{A}$ and $v$. From the properties of the operators $T_{1}$ and $K$ one can deduce the following a priori estimate for solutions to (38).

Theorem 12. Let $C_{A} \in C\left(\bar{B} ; \mathbb{C}_{m}\right), A \subset\{0,1, \ldots, m\}$, and $\left\|\sum_{A} C_{A}\right\|_{0} \leq M$ with $C M<1$. Let $F \in L_{p}\left(\bar{B} ; \mathbb{C}_{m}\right), f \in C^{\alpha}\left(\partial B ; \mathbb{C}_{m}\right)$. Then any solution to problem (38) satisfies

$$
\|w\|_{\alpha}+\|\bar{\partial} w\|_{p} \leq \gamma_{1}\|f\|_{\alpha}+\gamma_{2}\|F\|_{p}
$$

where the constants $\gamma_{1}$ and $\gamma_{2}$ only depend on $\alpha, p, C, M$.

This estimate can be used to solve nonlinear problems of the form

$$
\begin{gathered}
\bar{\partial} w=F(x, w) \quad \text { in } B, \\
(1+i x)(w-f(x, w))=0 \quad \text { on } \partial B
\end{gathered}
$$

(see [21]). Similarly another half-Dirichlet problem

$$
(1-i x)(w-f(x, w))=0 \quad \text { on } \partial B
$$

can be treated.

\section{References}

[1] H. Begehr, An approximation method for the Dirichlet problem of nonlinear elliptic systems in $\mathbb{R}^{r}$, Rev. Roumaine Math. Pures Appl. 27 (1982), 927-934.

[2] -, Boundary value problems for systems with Cauchy-Riemannian main part, in: Complex Analysis, Fifth Romanian-Finnish Seminar, Bucharest 1981, Lecture Notes in Math 1014, Springer, Berlin 1983, 265-279.

[3] - Boundary value problems for analytic and generalized analytic functions, in: Complex Analysis-Methods, Trends, and Applications, E. Lankau and W. Tutschke (eds.), Akad.Verlag, Berlin 1983, 150-165.

[4] - Remark on Hilbert's boundary value problem for Beltrami systems, Proc. Roy. Soc. Edinburgh 98A (1984), 305-310.

[5] H. Begehr and D. Q. Dai, Initial boundary value problem for nonlinear pseudoparabolic equations, Complex Variables Theory Appl., to appear.

[6] H. Begehr and R. P. Gilbert, Piecewise continuous solutions of pseudoparabolic equations in two space dimensions, Proc. Roy. Soc. Edinburgh 81A (1978), 153-173.

[7] -, - On Riemann boundary value problems for certain linear elliptic systems in the plane, J. Differential Equations 32 (1979), 1-14.

[8] - , - Boundary value problems associated with first order elliptic systems in the plane, in: Contemp. Math. 11, Amer. Math. Soc., 1982, 13-48. 
[9] H. Begehr and R. P. Gilbert, Pseudohyperanalytic functions, Complex Variables Theory Appl. 9 (1988), 343-357.

[10] H. Begehr and G. N. Hile, Nonlinear Riemann boundary value problems for a semilinear elliptic system in the plane, Math. Z. 179 (1982), 241-261.

[11] _, - , Riemann boundary value problems for nonlinear elliptic systems, Complex Variables Theory Appl. 1 (1983), 239-261.

[12] H. Begehr and G. N. Hile, Schauder estimates and existence theory for entire solutions of linear elliptic equations, Proc. Roy. Soc. Edinburgh 110A (1988), 101-123.

[13] H. Begehr and G. C. Hsiao, Nonlinear boundary value problems for a class of elliptic systems, in: Komplexe Analysis und ihre Anwendungen auf partielle Differentialgleichungen, Martin-Luther Universität, Halle-Wittenberg. Wissensch. Beiträge 1980, 90-102.

[14] - , - On nonlinear boundary value problems of elliptic systems in the plane, in: Ordinary and Partial Differential Equations, Proc. Dundee 1980, Lecture Notes in Math. 846, Springer, Berlin 1981, 55-63.

[15] —, - Nonlinear boundary value problems of Riemann-Hilbert type, in: Contemp. Math. 11, Amer. Math. Soc., 1982, 139-153.

[16] —, - The Hilbert boundary value problem for nonlinear elliptic systems, Proc. Roy. Soc. Edinburgh 94A (1983), 97-112.

[17] —, —, A priori estimates for elliptic systems, Z. Anal. Anwendungen 6 (1987), 1-21.

[18] H. Begehr and G.-C. Wen, The discontinuous oblique derivative problem for nonlinear elliptic systems of first order, Rev. Roumaine Math. Pures Appl. 33 (1988), 7-19.

[19] —, - A priori estimates for the discontinuous oblique derivative problem for elliptic systems, Math. Nachr. 142 (1989), 307-336.

[20] H. Begehr, G.-C. Wen and Z. Zhao, An initial and boundary value problem for nonlinear composite type systems of three equations, Math. Panonica 2 (1991), 49-61.

[21] H. Begehr and Z. Y. Xu, Nonlinear half-Dirichlet problems for first order elliptic equations in the unit ball of $\mathbb{R}^{m}(m \geq 3)$, Appl. Anal., to appear.

[22] L. Bers, Theory of Pseudoanalytic Functions, Courant Inst., New York 1953.

[23] L. Bers and L. Nirenberg, On a representation theorem for linear elliptic systems with discontinuous coefficients and its applications, in: Convegno Intern. Eq. Lin. Der. Parz. Trieste 1954, Edizioni Cremonese, Roma 1955, 111-140.

[24] B. Bojarski, A boundary value problem for a system of elliptic first order partial differential equations, Dokl. Akad. Nauk SSSR 102 (1955), 201-204 (in Russian).

[25] -, Homeomorphic solutions of Beltrami systems, ibid. 661-664 (in Russian).

[26] - On solutions of a linear elliptic system of differential equations in the plane, ibid. 871-874 (in Russian).

[27] —, Some boundary value problems for elliptic systems with two independent variables, Ph.D. thesis, Moscow Univ., 1955 (in Russian).

[28] —, Generalized solutions of a system of differential equations of the first order of elliptic type with discontinuous coefficients, Mat. Sb. 43 (85) (1957), 451-503 (in Russian).

[29] - Studies on elliptic equations in plane domains and boundary value problems of function theory, Habilitation thesis, Steklov Inst., Moscow 1960, 320 pp. (in Russian).

[30] - Subsonic flow of compressible fluid, Arch. Mech. Stos. 18 (1966), 497-519; also in: The Math. Problems in Fluid Mechanics, Polish Acad. Sci., Warszawa 1967, 9-32.

[31] —, Theory of generalized analytic vectors, Ann. Polon. Math. 17 (1966), 281-320 (in Russian).

[32] - Quasiconformal mappings and general structural properties of systems of non-linear equations elliptic in the sense of Lavrentiev, Symposia Math. 18 (1976), 485-499.

[33] B. Bojarski and I. Iw aniec, Quasiconformal mappings and non-linear elliptic equations in two variables. I, II, Bull. Acad. Polon. Sci. Sér. Sci. Math. Astronom. Phys. 22 (1974), 473-478; 479-484. 
[34] F. Brackx, R. Delanghe and F. Sommen, Clifford Analysis, Pitman, London 1982.

[35] D. Q. Dai, On an initial boundary value problem for nonlinear pseudoparabolic equations with two space variables, Complex Variables Theory Appl., to appear.

[36] R. Delanghe, F. Sommen and Z. Y. Xu, Half-Dirichlet problems for powers of the Dirac operator in the unit ball of $\mathbb{R}^{m}(m \geq 3)$, Bull. Soc. Math. Belg. Sér. B 42 (1990), 409-429.

[37] A. Douglis, A function-theoretic approach to elliptic systems of equations in two variables, Comm. Pure Appl. Math. 6 (1953), 259-289.

[38] A. Dzhuraev, Systems of Equations of Composite Type, Longman, Essex, 1989.

[39] F. D. Gakhov, Boundary Value Problems, Pergamon Press, Oxford 1966.

[40] D. Gilbarg and N. S. Trudinger, Elliptic Partial Differential Equations of Second Order, Springer, Berlin 1977.

[41] R. P. Gilbert and J. Buchanan, First Order Elliptic Systems: A Function Theoretic Approach, Acad. Press, New York 1983.

[42] R. P. Gilbert and G. N. Hile, Generalized hypercomplex function theory, Trans. Amer. Math. Soc. 195 (1974), 1-29.

[43] - - - Hypercomplex function theory in the sense of L. Bers, Math. Nachr. 72 (1976), $187-200$.

[44] W. Haak and W. Wendland, Lectures on Partial and Pfaffian Differential Equations, Pergamon Press, Oxford 1972.

[45] P. Henrici, Applied and Computational Complex Analysis, Vol. 3, Wiley, New York 1986.

[46] T. Iwaniec, Quasiconformal mapping problem for general nonlinear systems of partial differential equations, Symposia Math. 18 (1976), 501-517.

[47] V. N. Monakhov, Boundary-value problems with free boundaries for elliptic systems of equations, Amer. Math. Soc., Providence, R.I., 1983.

[48] N. I. Muskhelishvili, Singular Integral Equations, Noordhoff, Leyden 1977.

[49] W. Tutschke, Reduction of the problem of linear conjugation for first order nonlinear elliptic systems in the plane to an analogous problem for holomorphic functions, in: Lecture Notes in Math. 798, Springer, Berlin 1980, 446-455.

[50] —, Partielle Differentialgleichungen, Teubner, Leipzig 1983.

[51] I. N. Vekua, Generalized Analytic Functions, Pergamon Press, Oxford 1962.

[52] V. S. Vinogradov, On a boundary value problem for linear elliptic systems of first order in the plane, Dokl. Akad. Nauk SSSR 118 (1958), 1059-1062 (in Russian).

[53] —, On the boundedness of solutions of boundary value problems for linear elliptic systems of first order in the plane, ibid. 121 (1958), 399-402 (in Russian).

[54] - On some boundary value problems for quasilinear elliptic systems of first order in the plane, ibid., 579-581 (in Russian).

[55] -, A certain boundary value problem for an elliptic system of special form, Differentsial'nye Uravneniya 7 (1971), 1226-1234, 1341 (in Russian).

[56] G.-C. Wen and H. Begehr, Boundary Value Problems for Elliptic Equations and Systems, Longman, Essex 1990.

[57] W. Wendland, An integral equation method for generalized analytic functions, in: Constructive and Computational Methods for Differential and Integral Equations, Lecture Notes in Math. 430, Springer, Berlin 1974, 414-452.

[58] - On the imbedding method for semilinear first order elliptic systems and related finite element methods, in: Continuation Methods, Acad. Press, New York 1978, 277-336.

[59] —, Elliptic Systems in the Plane, Pitman, London 1979.

[60] J. Wloka, Funktionenanalysis und Anwendungen, de Gruyter, Berlin 1971.

[61] Z. X u, Boundary value problems and function theory for spin-invariant differential operators, thesis, State University of Ghent, Gent 1989. 